Rev. Biol. Trop., 47(1-2): 45-57, 1999

www.ucr.ac.cr www.ots.ac.cr www.ots.duke.edu

\title{
Crecimiento y mortalidad en juveniles de siete especies arbóreas en un bosque muy húmedo tropical intervenido de Costa Rica
}

Grace Sáenz, Bryan Finegan \& Manuel Guariguata

Centro Agronómico Tropical de Investigación y Enseñanza. Unidad de Manejo Bosques Naturales. CATIE 7170.

Apartado \# 68. Turrialba, Costa Rica. Fax: 556 2430. e-mail gsaenz@ catie.ac.cr

Received 11-V-1998. Corrected 1-XI-1998. Accepted 9-XII-1998.

\begin{abstract}
Diameter and height growth patterns, as well as the incidence of seedling (individuals e $0.3 \mathrm{~m}$ and $<1.5$ $\mathrm{m}$ in height) and sapling (individual e $1.5 \mathrm{~m}$ in height and $£ 9.9 \mathrm{~cm} \mathrm{DBH}$ ) mortality were studied for four years in a forest under two types of silvicultural intervention. The study is based on anual measurements of individuals from the two size categories marked one year after harvest. In the case of the seedlings, the parameters evaluated were: total height and diameter at $10 \mathrm{~cm}$ from the base. For the sapling category, the diameter at $1.30 \mathrm{~m}$ was also measured. The Clark and Clark methodology was used to evaluate the crown illumination index of all individuals. Seven tree species were studied: Cecropia (two species), Cecropia insignis Liebm. and Cecropia obtusifolia Bertol., Laetia procera (Poepp.) Eichler, Rollinia pittieri Saff, Apeiba membranacea Spruce ex Benth., Virola koschnyi Warb. y Virola sebifera Aubl. The Cecropia spp. seedlings showed the highest diameter and height growth rates, with annual mean increments in diameter of $7.8 \mathrm{~mm} /$ years and height of $188 \mathrm{~cm} / \mathrm{years}$. The $L$. procera saplings had the greatest diameter and height growth, with rates of $7.0 \mathrm{~mm} / \mathrm{year}$ and $86 \mathrm{~cm} / \mathrm{year}$, respectively. Only in the case of Cecropia spp. and V. sebifera are diameter and height growth significantly correlated with the crown light index. Seedlings had the highest mortality rates in all species except Cecropia spp. and $R$. pittieri, where the sapling mortality was greater.
\end{abstract}

Key words: Growth, mortality, seedling, saplings, tropical rain forest, natural regeneration, forest management.

El éxito del manejo de un bosque tropical depende en gran parte de la existencia de suficiente regeneración natural que asegure la sostenibilidad del recurso a través del tiempo. Los procesos que ocurren al caer un árbol son especialmente importantes para entender cambios en la estructura y dinámica de la comunidad arbórea (Hubbell y Foster 1986), sobre todo porque la diversidad de tamaños y formas de estas aperturas producen una diversidad de microambientes en luz, temperatura, humedad e intensidad y dirección de los vientos (Denslow 1980, Brokaw 1985), los cuales crean condiciones favorables para la regeneración natural de especies arbóreas. En el neotrópico, muchos de los estudios sobre regeneración natural de árboles se han efectuado en bosques sin manejo y se centran en la evaluación de la importancia de las aperturas del dosel en la composición y dinámica del rodal (Hartshorn 1978, Auspurger 1983, Brokaw 1985, Denslow 1987, Lieberman y Lieberman 1987, Popma y Bongers 1988, Connell 1989, Martínez-Ramos et al. 1989, Poulson y Platt 1989, Whitmore 1989, Welden et al. 1991, Clark y Clark 1992).

El aprovechamiento del bosque para madera puede causar un gran impacto sobre el rodal remanente, afectando la regeneración natural. Estudios realizados demuestran que la abundancia de especies con altos requerimientos de luz se incrementa con el aumento en la tasa de aperturas (Baur 1968, Jonkers 1987, 
Lamprecht 1990). Por otra parte algunos autores (Clark y Clark 1992), sugieren que si existe una diferenciación a nivel de especie en el crecimiento en respuesta a las aperturas. Desde el punto de vista silvicultural, es importante conocer el comportamiento de juveniles bajo condiciones de bosque aprovechado con el fin de evaluar la dinámica poblacional de especies comerciales en respuesta a cambios en el ambiente lumínico.

Este documento presenta los resultados de una investigación efectuada en la finca "La Tirimbina”, Costa Rica, en el cual se estudió durante cuatro años el crecimiento y supervivencia de brinzales y latizales de siete especies arbóreas en un bosque bajo manejo para la producción de madera.

\section{MATERIALES Y MÉTODOS}

Sitio de estudio: La investigación se realizó en la finca "La Tirimbina Rain Forest Center" localizada en la zona noreste de Costa Rica (100 24 N y 84006 W), en el cantón de Sarapiquí, Provincia de Heredia (Fig. 1). La elevación del lugar oscila entre los 150 y 200 m s.n.m. La temperatura media anual es de $25.3^{\circ} \mathrm{C}$ y la precipitación media anual es de 3883 mm (IMN 1992). Según la clasificación de Holdridge (1982) la zona corresponde a un bosque muy húmedo premontano transición a basal. El área posee 150 ha de bosque maduro aprovechado en varias ocasiones entre 1962 y 1990 (J.R. Hunter, comunicación personal). De esta superficie de bosque, el Centro Agronómico Tropical de Investigación y Enseñanza (CATIE) mantiene un área experimental de 29.2 ha bajo manejo con fines de producción de madera.

El paisaje del sitio es de colinas bajas formadas por la meteorización de coladas de lava andesítica. Los suelos presentes son Ultisoles, profundos, ácidos (pH ca.4.0), bien drenados y de baja fertilidad. La densidad aparente varía entre 0.58 y $0.66 \mathrm{~g} / \mathrm{cm}^{3}$, valores típicos para suelos de origen volcánico (Mata 1997).

Diseño experimental: El estudio se desarrolló en una superficie de 29.2 ha de bosque bajo manejo, dividida en tres bloques aleatorizados conformados por tres tratamientos. Dentro de cada tratamiento se ubicó una parcela de 1 ha con una faja de amortiguamiento de $40 \mathrm{~m}$ alrededor de la misma (Fig. 1); una descripción más detallada de los tratamientos se encuentra en Camacho y Finegan 1997; en este artículo no se incluye dicha descripción debido a que no es el objetivo del estudio comparar el efecto de los tratamientos sobre el crecimiento y la mortalidad de las especies estudiadas. Durante el período 1989 y 1990, se llevó a cabo un aprovechamiento selectivo en todo el área y en 1991 se aplicaron los tratamientos silviculturales (Fig. 1).

Para el presente estudio se marcaron brinzales (individuos e $0.30 \mathrm{~m}$ altura $\mathrm{y}<1.5 \mathrm{~m}$ altura) y latizales (individuos e $1.5 \mathrm{~m}$ altura y < $9.9 \mathrm{~cm}$ DAP) de siete especies arbóreas: dos especies de Cecropia: Cecropia insignis y $\mathrm{Ce}$ cropia obtusifolia (Cecropiáceas), Laetia procera (Flacourtiacae), Rollinia pittieri (Annonaceae), Apeiba membranacea (Tiliaceae), Virola sebifera y V. koschnyi (Myristicáceas), pertenecientes a grupos ecológicos contrastantes según la clasificación establecida antes por Finegan y Sabogal (1988): heliófita efímera (Cecropia spp.), heliófitas durables de crecimiento rápido (L. procera y $R$. pittieri), heliófitas durables de crecimiento regular (A. membranacea), esciófitas parciales ( $V$. koschnyi y V.sebifera). Los individuos de las especies de Cecropia spp., R. pittieri, L. procera y A. membranacea, se encontraron concentrados en las aperturas formadas por la intervención en dos de las parcelas del ensayo; en tanto que los individuos de las especies del género Virola, se localizaron en todo el área experimental.

El estudio se basa en mediciones periódicas de individuos de las dos categorías de tamaño, marcados un año después de la aplicación del aprovechamiento. Para la localización inicial de los individuos, se realizó un rastreo en las parcelas permanentes y los árboles de las especies de interés fueron identificados y marcados con placas de aluminio. En el caso de Cecropia spp., al inicio del experimento no se identificaron los individuos a nivel de especie 


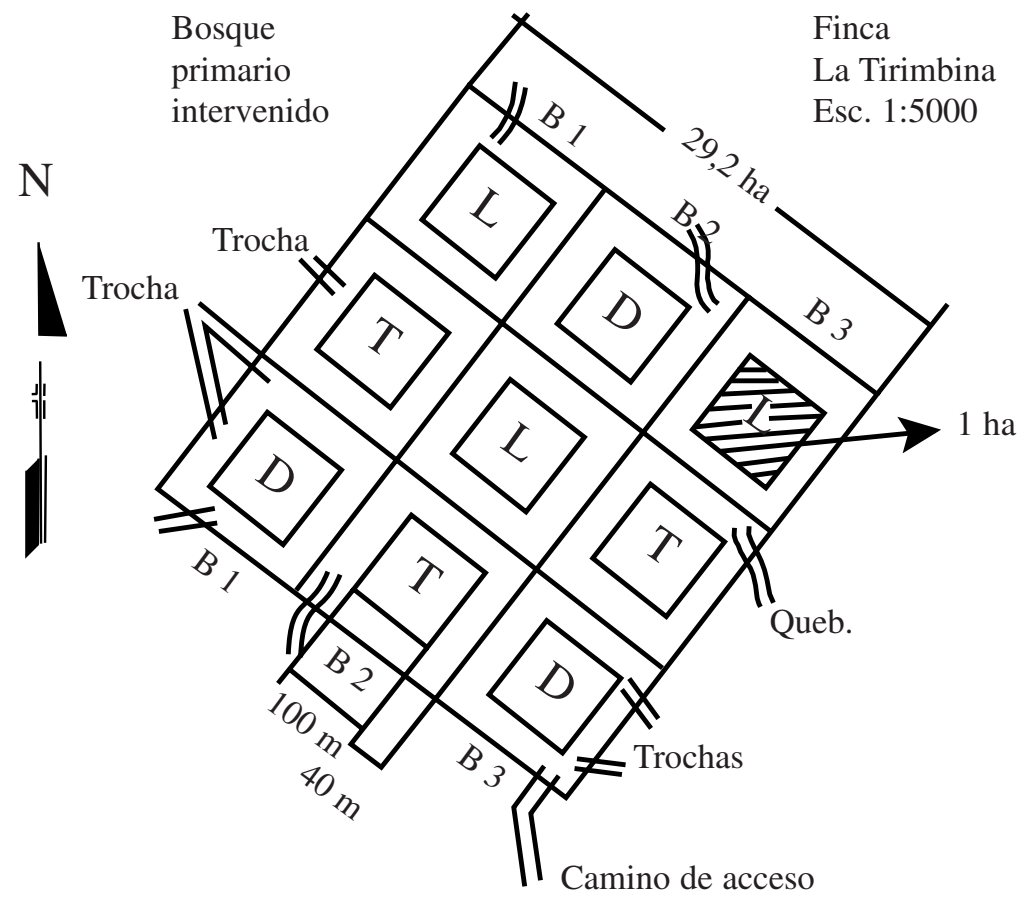

Fig. 1. Área Experimental de Manejo Finca "La Tirimbina Rain Forest Centre Inc." Código de tratamientos: L = Liberación + refinamiento parcial $\mathrm{T}=$ Testigo, $\mathrm{D}=$ Dosel protector.

ya que en ese momento se consideró suficiente con la identificación a nivel de género. Según Clark et al. (1993), en bosques cercanos (Estación Biológica La Selva) se encuentran dos especies de Cecropia (C. insignis y C. obtusifolia) cuyo comportamiento ecológico presenta diferencias a nivel de microambientes de luz, que en nuestro caso no fue posible discernir. Sin embargo, consideramos que la información obtenida de la comparación entre éstas especies con otras supuestamente más tolerantes a la sombra ayuda a comparar el comportamiento demográfico de nuestras especies en estudio.

Los parámetros evaluados fueron en el caso de los brinzales: la altura total $(\mathrm{cm})$ y el diámetro a $10 \mathrm{~cm}$ de la base $(\mathrm{mm})$ medido con un calibrador (precisión de $0.1 \mathrm{~mm}$ ). Cuando el individuo pertenecía a la categoría de latizal se midió además el diámetro a $1.30 \mathrm{~m}$. Se midió también el índice de iluminación de la copa utilizando la metodología propuesta por Clark y Clark (1992) pues permite obtener una medición fácil y directa basada en la estructura de la vegetación por todos los lados y arriba del árbol: (1) solamente luz indirecta, (1.5) baja luz directa oblicua lateral, (2) mediana luz directa oblicua lateral, (2.5) alta luz directa oblicua lateral, (3) alguna luz directa vertical, (4) luz vertical plena, (5) copa completamente expuesta.

Para L. procera, A. membranacea, $R$. pittieri y Cecropia spp., el estudio se realizó durante un período de cuatro años (diciembre 1991 - diciembre 1995). En el caso de las dos especies de Virola, los datos fueron registrados durante un período de 3 años (diciembre 1992-diciembre 1995). En ambos casos, las mediciones fueron realizadas cada tres meses durante el primer año y anualmente durante los dos últimos años.

Los datos de mortalidad de todas las especies fueron registrados a partir de diciembre de 1992. Cuando algún individuo no se encontró se hizo una anotación y si al año siguiente de nuevo no se logró localizar se anotó como muerto (indicando como año de muerte la medición anterior). La tasa anual de mortalidad se 
calculó utilizando el modelo logarítmico descrito por Swaine et al. (1987):

$$
\mathrm{m}=100\left(\log _{\mathrm{e}} \mathrm{no}-\log _{\mathrm{e}} \mathrm{n} 1\right) / \mathrm{t}
$$

en donde no= número de individuos al inicio de la medición; $\mathrm{n} 1$ = número de individuos al final del período de medición y $\mathrm{t}=$ tiempo en años.

En el caso de $R$. pittieri y Cecropia spp. no se realizaron análisis de los datos de la categoría de latizal debido al escaso número de individuos vivos de estas especies al final del período de investigación, por lo tanto, para estas dos especies en los Cuadros no se reportan datos en dicha categoría.

Para el análisis estadístico de la información fueron aplicadas pruebas no paramétricas. Para determinar diferencias de crecimiento y de índices de iluminación entre especies se aplicó la prueba U de Mann-Whitney. Por último, para estudiar la posible interdependencia del índice de iluminación de copa con el incremento en altura y en diámetro en las distintas categorías de tamaño, se realizaron análisis de correlación con la prueba de Spearman.

\section{RESULTADOS}

Crecimiento en diámetro y altura: Los valores de la mediana, máximos y mínimos del incremento periódico e incremento total en diámetro y altura se presentan en el Cuadro 1 y 2. En el caso de los individuos de la categoría de brinzal, la mediana del incremento para el período total en diámetro osciló entre 7.8 $\mathrm{mm} / \mathrm{año} \mathrm{y} 1.5 \mathrm{~mm} / \mathrm{año}$, en tanto que el incremento en altura para ese mismo período presentó valores entre $188 \mathrm{~cm} /$ año y $18 \mathrm{~cm} / \mathrm{año}$. Para ambas variables los mayores valores se obtuvieron en las especies de Cecropia y los valores mínimos en la especie $V$. sebifera. Para algunas de las especies se encontraron los mayores incrementos diamétricos y en altura durante el período 1991-1992 (inmediatamente después del aprovechamiento). Posteriormente en los período 1992-1994 y 94-95 se observó una disminución en el crecimiento (Cuadro 1 y
2). La excepción a esta tendencia fue A. membranacea que presentó un aumento en su crecimiento en el período 1994-1995 y las dos especies de Virola, las cuales mostraron medianas de crecimiento diamétrico y en altura mayores durante ese mismo período.

En la categoría de latizal se encontró que la mediana de crecimiento diamétrico para el período total osciló entre $7.0 \mathrm{~mm} / \mathrm{año} \mathrm{y} 2.7 \mathrm{~mm} / \mathrm{año} \mathrm{y}$ para el incremento en altura entre $86 \mathrm{~cm} / \mathrm{año} \mathrm{y} 40$ $\mathrm{cm} / \mathrm{año}$, obteniéndose estos valores para las especies L. procera y A. membranacea, respectivamente (Cuadro 1 y 2). Todas las especies mostraron su mayor incremento diamétrico y en altura durante el primer período de medición, disminuyendo paulatinamente a través del tiempo.

Comparación del crecimiento entre especies: Se efectuaron todas las posibles comparaciones pareadas del incremento diamétrico anual del período total entre las diferentes especies (Cuadro 3). Las diferencias fueron más evidentes entre las especies supuestamente más demandantes de luz con respecto a aquellas más tolerantes. Se observó en la categoría de brinzal que Cecropia spp. creció significativamente más que las demás especies con excepción de L. procera. Así mismo, L. procera, presentó un crecimiento diamétrico significativamente mayor que las dos especies de del género Virola, mientras que $R$. pittieri, mostró también un crecimiento diamétrico significativamente mayor que los brinzales de V. sebifera (Cuadro 3). En la categoría de latizal no fue posible efectuar comparaciones incluyendo Cecropia spp. y R. pittieri, debido al escaso número de individuos, sin embargo, se logra observar que las diferencias son menos obvias. Ya no se encontraron diferencias significativas entre $L$. procera con respecto a $V$. sebifera ni $V$. koschnyi e inclusive, A. membranacea muestra en esa categoría, un crecimiento en diámetro significativamente menor con respecto a esas últimas dos especies (Cuadro 3).

De igual se efectuaron todas las comparaciones posibles con el incremento anual en altura para el período total entre las diferentes especies. En la categoría de brinzal Cecropia 


\section{CUADRO 1}

Valores de la mediana, máximas y mínimas del incremento periódico y para el incremento total en diámetro por especie ( mm/año) para la categoría de brinzal y latizal, para individuos supervivientes durante todo el período.

\begin{tabular}{|c|c|c|c|c|c|c|c|c|c|}
\hline & & & & & to diar & $\mathrm{o} \mathrm{mn}$ & & & \\
\hline & & & & & & & & & \\
\hline Cod. Esp & Período & $\mathrm{n}$ & Med & Max & Min & $\mathrm{n}$ & Med & $\operatorname{Max}$ & Min \\
\hline Cspp. & $91-92$ & 6 & 18.1 & 28.6 & 6.2 & - & & & \\
\hline & $92-94$ & & 5.2 & 8.9 & 2.4 & & & & \\
\hline & $94-95$ & & 1.5 & 9.0 & 0 & & & & \\
\hline & Total & & 7.8 & 12.1 & 2.7 & & & & \\
\hline $\mathrm{Rp}$ & $91-92$ & 6 & 8.3 & 18.9 & 4.2 & - & & & \\
\hline & $92-94$ & & 2.0 & 4.1 & 1.0 & & & & \\
\hline & 94-95 & & 0.5 & 4.0 & 0.5 & & & & \\
\hline & Total & & & & & & & & \\
\hline $\mathrm{Lp}$ & $91-92$ & 22 & 8.9 & 23.6 & 1.8 & 50 & 13.0 & 38.0 & 0.90 \\
\hline & $92-94$ & & 2.2 & 15.1 & -0.8 & & 5.1 & 31.2 & -1.0 \\
\hline & $94-95$ & & 2.0 & 18.0 & 0 & & 4.0 & 25.0 & -3.0 \\
\hline & $94-95$ & & 4.1 & 14.4 & 1.2 & & 7.0 & 26.0 & 0.6 \\
\hline & Total & & & & & & & & \\
\hline $\mathrm{Am}$ & $91-92$ & 15 & 6.0 & 22.3 & 0.4 & 30 & 5.0 & 40 & 0.5 \\
\hline & $92-94$ & & 0.8 & 8.5 & -0.6 & & 1.6 & 27.4 & -1.4 \\
\hline & 94-95 & & 1.0 & 5.0 & -1.0 & & 1.0 & 15.0 & -1.0 \\
\hline & 94-95 & & 2.4 & 6.4 & 0.4 & & 2.7 & 24.7 & 0.2 \\
\hline & Total & & & & & & & & \\
\hline Vk & $92-94$ & 9 & 0.8 & 3.5 & -0.3 & 14 & 8.8 & 23.0 & 1.8 \\
\hline & 94-95 & & 4.0 & 10.0 & 1.0 & & 3.0 & 14.0 & 0 \\
\hline & $94-95$ & & 1.6 & 4.9 & 4.9 & & 6.7 & 17.0 & 2.2 \\
\hline & Total & & & & & & & & \\
\hline Vs & $92-94$ & 15 & 0.9 & 3.2 & -1.2 & & 6.5 & 23.8 & 0.4 \\
\hline & 94-95 & & 3.0 & 21.0 & -1.0 & 27 & 5.0 & 18.0 & -1.0 \\
\hline & $94-95$ & & 1.5 & 4.1 & 0.5 & & 6.9 & 17.1 & 1.2 \\
\hline
\end{tabular}

Código de especies: (Cspp) Cecropia spp., (Rp) R. pittieri, (Lp) L. procera, (Am) A. membranacea, (Vk) V. koschnyi, (Vs) V. sebifera. 
CUADRO 2

Valores de la mediana, máximas y mínimas del incremento periódico y para el incremento total en altura por especie ( cm/año) para la categoría de brinzal y latizal, para individuos supervivientes durante todo el período.

\begin{tabular}{|c|c|c|c|c|c|c|c|c|c|}
\hline & & & & & to dian & $\mathrm{o} \mathrm{mm}$ & & & \\
\hline & & & & & & & & & \\
\hline Cod. Esp & Período & $\mathrm{n}$ & Med & Max & Min & $\mathrm{n}$ & Med & Max & Min \\
\hline Cspp. & $91-92$ & 6 & 216 & 385 & 95 & - & & & \\
\hline & $92-94$ & & 104 & 173 & 21 & & & & \\
\hline & $94-95$ & & 33 & 135 & -33 & & & & \\
\hline & $94-95$ & & 188 & 178 & 53 & & & & \\
\hline & Total & & & & & & & & \\
\hline $\mathrm{Rp}$ & $91-92$ & 6 & 151 & 217 & 62 & & & & \\
\hline & $92-94$ & & 47 & 97 & -43 & & & & \\
\hline & $94-95$ & & 4 & 95 & -51 & & & & \\
\hline & $94-95$ & & 38 & 103 & 7 & & & & \\
\hline & Total & & & & & & & & \\
\hline Lp & $91-92$ & 22 & 107 & 34 & 50 & 50 & 130 & 380 & 90 \\
\hline & $92-94$ & & 49 & 170 & 6 & & 70 & 256 & -20 \\
\hline & $94-95$ & & 39 & 240 & -133 & & 52 & 293 & -186 \\
\hline & $94-95$ & & 64 & 198 & 18 & & 86 & 225 & 13 \\
\hline & Total & & & & & & & & \\
\hline $\mathrm{Am}$ & $91-92$ & 15 & 2 & 85 & -33 & 30 & 87 & 354 & -17 \\
\hline & $92-94$ & & 18 & 88 & -39 & & 13 & 227 & -174 \\
\hline & $94-95$ & & 31 & 86 & -44 & & 34 & 160 & -134 \\
\hline & $94-95$ & & & 100 & -10 & & 40 & 212 & -0.18 \\
\hline & Total & & & & & & & & \\
\hline Vk & $92-94$ & 9 & 20 & 30 & -4 & 14 & 67 & 194 & -14 \\
\hline & $94-95$ & & 41 & 11 & 0 & & 18 & 182 & -45 \\
\hline & $94-95$ & & 25 & 54 & 1 & & 57 & 150 & 6 \\
\hline & Total & & & & & & & & \\
\hline Vs & $92-94$ & 15 & 17 & 74 & -3 & 27 & 67 & 202 & -82 \\
\hline & $94-95$ & & 8 & 104 & -1 & & 3 & 224 & $-1,53$ \\
\hline & $94-95$ & & 18 & 90 & 3 & & 62 & 150 & 0 \\
\hline
\end{tabular}

Ver código de especies en el Cuadro 1. 
CUADRO 3

Resultados de la prueba Mann-Whitney aplicada al incremento anual en diámetro y altura durante el período total por categoría de regeneración

$\begin{array}{llllll}\text { Cspp. } & \text { Rp } & \text { Lp } & \text { Am } & \text { Vk }\end{array}$

\begin{tabular}{|c|c|c|c|c|c|}
\hline \multicolumn{6}{|l|}{ Diámetro } \\
\hline $\mathrm{Rp}$ & 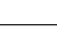 & $\mathrm{ns}$ & $\mathrm{ns}$ & $\mathrm{ns}$ & $>* *$ \\
\hline Lp & & 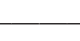 & & $>* *$ & $>* * *$ \\
\hline $\mathrm{Am}$ & & $<* * *$ & 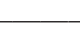 & $\mathrm{ns}$ & $\mathrm{ns}$ \\
\hline $\mathrm{Vk}$ & & $\mathrm{ns}$ & $>* * *$ & 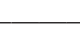 & $\mathrm{ns}$ \\
\hline Vs & & ns & $>* * *$ & $\mathrm{~ns}$ & 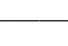 \\
\hline Altura & & & & & \\
\hline Cspp. & $>*$ & $\mathrm{~ns}$ & $>* * *$ & $>* * *$ & $>* *$ \\
\hline $\mathrm{Rp}$ & 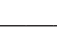 & $\mathrm{ns}$ & $\mathrm{ns}$ & $\mathrm{ns}$ & $\mathrm{ns}$ \\
\hline $\mathrm{Lp}$ & & & $>* * *$ & $>* * *$ & $>* * *$ \\
\hline Am & & $<* * *$ & 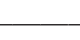 & ns & ns \\
\hline Vk & & $<*$ & ns & 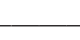 & $\mathrm{ns}$ \\
\hline Vs & & $<* *$ & $>* *$ & $\mathrm{~ns}$ & 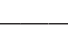 \\
\hline
\end{tabular}

Probabilidad de la prueba Mann-Whitney $* 0.05>\mathrm{p}>0.01, * * \mathrm{p}<0.01, * * * \mathrm{p}<0.001$

Los valores arriba de las diagonales corresponden a la categoría de brinzal y por debajo de éstas, los valores corresponden a la categoría de latizal. Los signos $(>)$ o $(<)$ se refieren al mayor o menor crecimiento de la especie ubicada en la fila con respecto a la especie ubicada en la columna. Ver código de especies en el Cuadro 1.

spp. mostró un mayor crecimiento con respecto a las demás especies, con excepción de $L$. procera (Cuadro 3). Así mismo, se encontraron diferencias altamente significativas entre $L$. procera con relación a $A$. membranacea y las dos especies de Virola (Cuadro 3). En la categoría de latizal, L. procera (a diferencia de lo ocurrido en el análisis del incremento diamétrico) mantiene un crecimiento significativamente mayor que $A$. membranacea y las dos especies de Virola (Cuadro 3).

Condiciones de iluminación de la regeneración natural: En la primer evaluación de micrositios de luz (tres años después del aprovechamiento), la mayoría de las especies presentaron entre el $40 \%$ y el $73 \%$ de sus individuos con índices de iluminación de copa de 2.5 (luz lateral alta; Cuadro 4). Según categorías de tamaño, la mediana del índice de ilumina- ción de copa para los brinzales de la mayoría de las especies fue 2.5 (luz lateral alta), con excepción de las dos especies de Virola cuya mediana fue de 2.0 (luz lateral media) y presentaron además los mínimos más extremos en la mediana de iluminación (Fig 2a). Además, se observa que el ámbito de iluminación aumenta en latizales para todas las especies, alcanzando valores máximos de 3.0 y 4.0 (Fig 2b).

Se detectaron, en el caso de los brinzales, diferencias significativas de iluminación entre Cecropia spp. con respecto a $V$. sebifera y $V$. koschnyi, obteniendo Cecropia spp. en ambos casos, la mayor iluminación de copa (Cuadro 5). Además, fueron significativamente diferentes las medianas de iluminación de la especie $V$. sebifera (menor) con respecto a $L$. procera y $A$. membranacea (mayor) (Cuadro 5). Así mismo, se lograron encontrar diferencias en el índice de iluminación de copa entre $V$. koschnyi y $A$. 
CUADRO 4

Estadísticas del índice de iluminación de copa por especie y categoría de regeneración

\begin{tabular}{llllllll} 
Categoría & Estadist. & Cspp. & Rp & Lp & Am & Vk & Vs \\
Brinzal & $\mathrm{N}$ & 6 & 6 & 22 & 15 & 9 & 15 \\
& Mediana & 2.5 & 2.5 & 2.5 & 2.5 & 2.0 & 2.0 \\
\multirow{3}{*}{ Latizal } & Rango & $2.0-3.0$ & $2.0-2.5$ & $1.5-3.0$ & $2.0-2.5$ & $1.5-2.5$ & $1.5-2.5$ \\
& $\mathrm{~N}$ & & & 50 & 30 & 14 & 27 \\
& Mediana & & & 2.5 & 2.5 & 2.0 & 2.5 \\
& Rango & & & $1.5-4.0$ & $1.5-3.0$ & $1.5-3.0$ & $2.0-3.0$
\end{tabular}

Ver código de especies en el Cuadro 1.
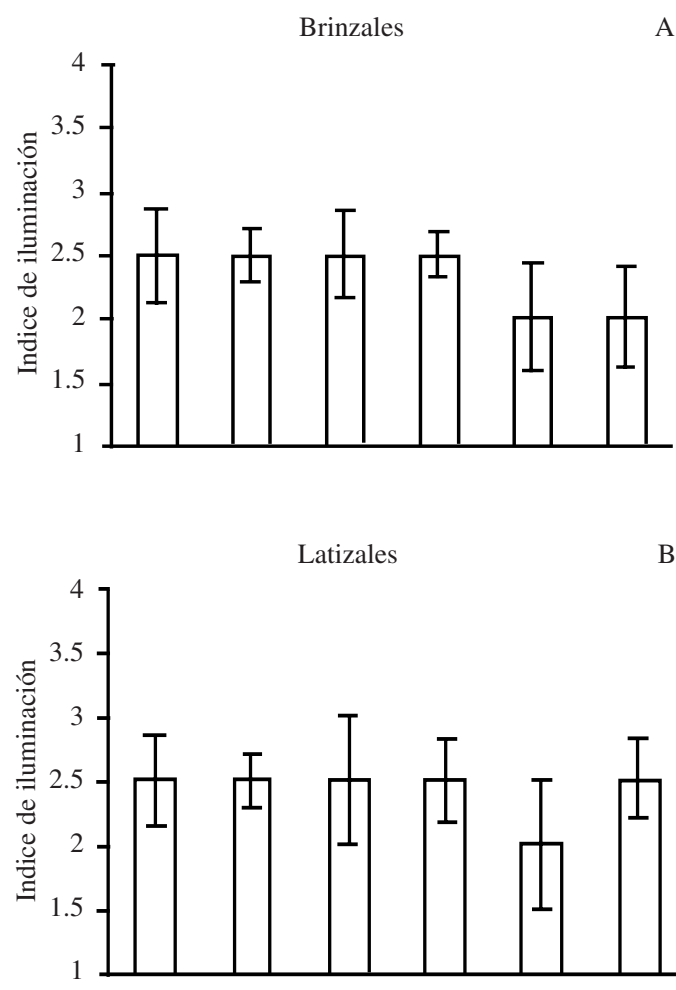

Fig. 2 a,b. Medianas del "Indice de Iluminación de Copa" por especie y categoría de regeneración; las líneas verticales representan la desviación estandar. Ver códigos de especies en el Cuadro 1.

membranacea.. En el caso de los latizales, el número de comparaciones posibles disminuye. Sin embargo, se detectaron diferencias estadísticamente mayores entre L. procera con $V$. koschnyi y A. membranacea.
Efecto de la exposición de copa sobre el crecimiento: En el caso de los brinzales, se mostró una correlación positiva y significativa entre el índice de iluminación y el incremento en diámetro y altura para las especies: Cecropia spp. y para V. sebifera (Cuadro 6). En el caso del género Cecropia, se encontraron coeficientes de correlación muy altos tanto para el incremento diamétrico como para el incremento en altura. Para el caso de V. sebifera, los coeficientes de correlación fueron un poco menores, pero aún arriba del 50\% y el nivel de significancia fue más alto en el caso del incremento en altura. A nivel de latizal, V. sebifera sigue correlacionando su crecimiento con el índice de iluminación de copa, aunque los valores del índice de correlación son un poco menores que los presentados por esta misma especie a nivel de brinzal (Cuadro 6). Además $L$. procera presentó una correlación altamente significativa y positiva con ambas variables.

Mortalidad: La mortalidad fue mayor en los individuos de la categoría de brinzal que en los latizales (Cuadro 7). Excepciones en este comportamiento lo presentaron Cecropia spp. y $R$. pittieri, especies que tuvieron una mortalidad mayor en la categoría de latizal. Las mayores tasas de mortalidad se presentaron en $\mathrm{Ce}$ cropia spp., L. procera y $R$. pittieri. Cabe destacar el hecho de que las especies clasificadas $a$ priori como esciófitas (V. sebifera y V. koschn$y i)$ no presentaron mortalidad en ninguna de las categorías de tamaño durante todo el estudio. 


\section{CUADRO 5}

Comparaciones entre especies del índice de iluminación de copa por especie y categoría de regeneración (prueba de Mann-Whitney)

$\begin{array}{lc}\text { Especie } & \text { Prob. } \\ \text { Brinzales } & \\ \text { Cspp. vs Vs } & * * \\ \text { Cspp. vs Vk } & * \\ \text { Lp vs Vs } & * * * \\ \text { Am vs Vs } & * * * \\ \text { Am vs Vk } & * \\ \text { Latizales } & \\ \text { Lp vs Vk } & * * \\ \text { Lp vs Am } & *\end{array}$

Probabilidad de la prueba Mann -Whitney * $0.05>\mathrm{p}>0.01, * * \mathrm{p}<0.01 * * * \mathrm{p}<0.001$

La especie que aparece de primera en cada una de las comparaciones es la que presentó una mediana de exposición de copa mayor. Ver código de especies en el Cuadro 1.

\section{CUADRO 6}

Resultados del análisis de correlación ( $r$ de Spearman) entre el índice de iluminación de copa y el crecimiento en diámetro y altura

Incr. DiamétricoCoef. corr $\quad$ p $\quad$ Incr. AlturaCoef. corr $\quad p$

Brinzales

Latizales

Lp $\quad 0.66$

$\begin{array}{ll}\text { Vs } & 0.47\end{array}$
0.57

0.90
*

$* *$

$* * *$

**
0.64

0.83

0.59

0.48
$* *$

$*$

***

$* *$

Probabilidad de la prueba de correlación de Spearman * $0.05>\mathrm{p}>0.01 * * \mathrm{p}<0.01$

Ver código de especies en el Cuadro 1. 


\section{CUADRO 7}

Tasa anual exponencial de mortalidad de brinzales y latizales durante los diferentes períodos

\begin{tabular}{lccc} 
Especie & \multicolumn{3}{c}{ Tasa anual exponcial de mortalidad } \\
& $\mathrm{n}_{0}$ & $92 / 94$ & $94 / 95$ \\
Brinzales & & & \\
Cspp. & 32 & 69 & 29 \\
Rp & 17 & 22 & 60 \\
Lp & 110 & 59 & 44 \\
Am & 30 & 23 & 24 \\
Vk & 9 & 0 & 0 \\
Vs & 15 & 0 & 0 \\
& & & \\
Latizales & & 80 & 09 \\
Cspp. & 10 & 50 & 13 \\
Rp & 11 & 10 & 10 \\
Lp & 80 & 10 & 0 \\
Am & 40 & 0 & 0 \\
Vk & 14 & 0 & \\
Vs & 27 & &
\end{tabular}

Ver códigos de especies en el Cuadro1.

\section{DISCUSIÓN}

Iluminación de micrositios ocupados por la regeneración natural: En cuanto a las condiciones de iluminación, observamos que las medianas del índice de iluminación encontradas en juveniles en nuestro estudio son similares a los datos reportados por Clark y Clark (1992) y Clark et al. (1993), también para juveniles de seis especies (diferentes a las evaluadas en el presente documento) en un bosque no intervenido estructuralmente semejante al sitio de estudio. Estos autores reportan tanto para especies pioneras como para tolerantes a la sombra, medianas de índice de iluminación entre 2 y 2.5 , indicando con ello que la gran mayoría de los individuos tanto de la categoría de brinzal como de latizal reciben luz lateral alta y son pocos los individuos que reciben luz directa por arriba.

Lo anterior induce a pensar que si bien es cierto la intervención del bosque modificó en alguna medida las condiciones originales de iluminación del mismo, parece ser que los tratamientos aplicados no fueron tan drásticos como para producir, en los estratos más bajos del bosque, cambios radicales en las condiciones de luz, comparadas con las encontradas en el bosque sin intervención.

Las diferencias entre especies a nivel de micrositio lumínico no fueron tan evidentes en latizales pero sí en brinzales. Este hecho permite inferir que a nivel de brinzal, las condiciones de iluminación de las especies estudiadas parecen ser relativamente diferenciables durante el período de estudio (cuatro años). En el caso de la categoría de latizal, las especies se encontraron en un ámbito más amplio de condiciones lumínicas, produciéndose un mayor traslape de micrositios. Lo anterior implica que a mediano plazo los latizales de las especies estudiadas pueden considerarse como "generalistas" (Welden et al. 1991). Sin embargo estas tendencias podrían cambiar en clases de tamaño mayores que las aquí estudiadas o con seguimiento a largo plazo.

Patrones de crecimiento e iluminación de la copa: De manera general encontramos que el crecimiento presentó una relación positiva con el tamaño inicial de los individuos, siendo mayores los incrementos medianos en diámetro y altura en la categoría de latizal. Un comportamiento similar fue reportado por 
Clark y Clark (1992) en La Selva. En nuestro caso, lo anterior fue evidente para todas las especies, pero fue especialmente notorio en el caso de Virola, en donde se lograron incrementos diamétricos en la categoría de latizal aproximadamente tres veces mayores que los obtenidos para los brinzales.

Otro aspecto importante es la clara diferencia en las tasas de crecimiento entre las especies, tanto en diámetro como en altura. El mayor crecimiento demostrado por Cecropia, $L$. procera y $R$. pittieri, en relación con $A$. membranacea y las dos especies de Virola podría deberse tanto a características fisiológicas inherentes a las especies, como también a la desventaja inicial (menor iluminación) que presentaban los brinzales de algunas de estas especies en relación con las otras. Esto explicaría en parte el hecho de que los latizales de Virola, cuando se encuentran bajo condiciones de luz semejante a las de otras especies supuestamente más demandantes de luz, logran alcanzar tasas de crecimiento diamétrico similares, aunque su crecimiento en altura fue significativamente inferior. En este sentido otros estudios han encontrado resultados en donde algunas especies tolerantes a la sombra han demostrado tasas de crecimiento similares a las especies pioneras (Manokaran y Kochummen 1987), lo cual podría reafirmar el planteamiento anterior.

En cuanto al efecto de la iluminación sobre el crecimiento de las especies estudiadas, se encontró correlación positiva entre ambas variables únicamente en dos de las siete especies estudiadas. Sin embargo cuando se encontró tal correlación, ésta fue significativa tanto para el incremento diamétrico como en altura. Por su parte, Clark et al. (1993) encontraron que los juveniles de especies arbóreas correlacionaron significativamente su crecimiento en diámetro y altura con el índice de iluminación de copa. Lo anterior sugiere que en bosques sin manejo, el crecimiento de los individuos juveniles es más limitado por las condiciones de luz que en el caso de un bosque bajo manejo. Tal situación podría explicar el menor número de correlaciones encontradas en nuestro estudio y reafirma el hecho de en el caso de los bosque en estado inalterado las aperturas del dosel podrían tener un papel más determinante.

Demografía: Se encontró que la tasa de mortalidad de la mayoría de las especies estudiadas fueron mayores que las reportadas en otros estudios (Lieberman y Lieberman 1987, Nicolson citado por Swaine et al. 1987, Clark y Clark 1992). Estos últimos autores señalan para seis especies no pioneras (en bosque sin intervenir), porcentajes de mortalidad entre 2.7 y $19 \%$ para la clase $£ 1.5 \mathrm{~cm}$ de diámetro, porcentajes que van disminuyendo al aumentar la clase de tamaño de los individuos (alcanzando valores entre 0 y $1.7 \%$ para individuos de la categoría $>4-10 \mathrm{~cm}$ de diámetro). En el presente artículo se encontró porcentajes de mortalidad un poco mayores en las especies más demandantes de luz, alcanzando en la categoría de brinzal entre 0 y $60 \%$ y en la categoría de latizal entre 0 y $69 \%$. Sin embargo las especies de Virola mostraron durante nuestro estudio una tasa de supervivencia del $100 \%$, tanto en la categoría de brinzal como de latizal, lo cual coincide con las características de las especies tolerantes a la sombra. Resultados semejantes fueron encontrados por Clark y Clark (1992). Las mayores tasas de mortalidad encontradas para Cecropia spp. parece ser consecuencia de la competencia entre individuos que crecen en claros (especialmente especies pioneras).

Otro aspecto que debe mencionarse es que las tasas de mortalidad mayores, así como también las mayores tasas de crecimiento, se obtuvieron en $L$. procera, $R$. pittieri y Cecropia spp. En tanto, A. membranacea y las dos especies de Virola presentaron tasas de supervivencia más altas pero asociadas a menores tasas de crecimiento.

Con base en los argumentos mencionados, consideramos que a nivel juvenil, la agrupación estricta de especies en gremios ecológicos es operacionalmente difícil, siendo preferible adoptar una posición más flexible y elaborar un historial de las características de micrositio de las diferentes especies que permita conocer más acerca de sus patrones de crecimiento y supervivencia a través del tiempo. Tal posición 
parece más acertada considerando que "rara vez será factible caracterizar el comportamiento de una especie desde semilla hasta el adulto con un término como "tolerante a la sombra" o “dependiente de claros" (Clark y Clark 1987).

\section{RESUMEN}

Se estudiaron por un período de cuatro años los patrones de crecimiento en diámetro y altura, así como la mortalidad de brinzales (individuos e $0.30 \mathrm{~m}$ altura y < $1.5 \mathrm{~m}$ altura) y latizales (individuos e $1.5 \mathrm{~m}$ altura y $£ 9.9$ cm DAP) en un bosque sometido a dos tipos de intervención. El estudio se basa en mediciones anuales de individuos de las dos categorías de tamaño, marcados un año después de la aplicación del aprovechamiento. Siete especies arbóreas fueron estudiadas: Cecropia (dos especies) Cecropia insignis y Cecropia obtusifolia, Laetia procera, Rollinia pittieri, Apeiba membranacea, Virola koschnyi. y Virola sebifera. A nivel de brinzal las especie que presentaron el mayor crecimiento en diámetro y en altura fueron las especies del género Cecropia, mientras que en la categoría de latizal, fue L. Procera. El crecimiento en diámetro y en altura se correlacionó significativamente con el índice de iluminación de copa, únicamente en el caso de las dos especies de Cecropia y V. sebifera. Las tasas de mortalidad más altas se encontraron en los brinzales.

\section{REFERENCIAS}

Anónimo. 1992. Apuntes climatológicos estación La Selva, período 1959-1986. Instituto Meteorológico Nacional. San José, Costa Rica. s.p.

Auspurger, C.K. 1983. Seed dispersal of the tropical tree, Platypodium elegans, and the escape of its seedlings from fungal pathogens. J. Ecol. 71: 759-771.

Baur, G. 1968. The ecological basis of rain forest management. FAO. Sidney, Australia. 499 p.

Brokaw, N.V. \& S.M. Scheiner. 1989. Species composition in gaps and structure of a tropical forest. Ecology 70: 538-541.

Camacho, M. \& B. Finegan. 1997. Efectos del aprovechamiento forestal y el tratamiento silvicultural en un bosque húmedo del noreste de Costa Rica: el crecimiento diamétrico con énfasis en el rodal comercial. Colección Manejo Diversificado de Bosques Naturales. CATIE, Cartago, Costa Rica. 38 p.

Clark, D.B. \& D.A. Clark. 1987. Population ecology and microhabitat distribution of Dipteryx panamensis, a neotropical rain forest emergent tree. Biotropica 19: 236-244.

Clark, D.A. \& D.B. Clark. 1992. Life history diversity of canopy and emergent trees in a neotropical rain forest. Ecol. Monogr. 62: 315-344.

Clark, D.A., D.B. Clark \& P.M. Rich. 1993. Comparative analisys of microhabitat utilization by saplings of nine tree species in neotropical rain Forest. Biotropica 254: 397-407.

Connell, J.H. 1989. Some processes affecting the species composition in forest gaps. Ecology 70: 560-562.

Denslow, J.S. 1980. Gap partitioning among tropical rainforest trees. Biotropica 12 (Suppl): 47-55.

Denslow, J.S. 1987. Tropical rain forest gaps and tree species diversity. Annu. Rev. Ecol. Syst. 18: 431-451.

Finegan, B. \& C. Sabogal. 1988. El desarrollo de sistemas de producción sostenible en bosques tropicales húmedos de bajura: un estudio de caso en costa rica. Chasqui 17: 1-24.

Hartshorn, G.S. 1978. Tree falls and tropical forest dynamics, p. 617-638. In P.B. Tomlinson (eds). Tropical trees as living systems. Cambridge University, Londres.

Holdridge, L. 1982. Ecología basada en zonas de vida. IICA, San José, Costa Rica. 216p.

Hubbell, S. P. \& R.B. Foster. 1986. Canopy gaps and the dynamics of a tropical forest, p. 77-96. In M.J. Crawley (ed.). Plant ecology. Blackwell Scientific, Oxford.

Jonkers, W.B. 1987. Vegetation structure logging damage and silviculture in a tropical rain forest in Suriname. Agricultural University, Wageningen, Holanda. 172 p.

Lamprecht, H. 1990. Silvicultura en los trópicos: los ecosistemas forestales en los bosques tropicales y sus especies arbóreas; posibilidades y métodos para un aprovechamiento sostenido. Deutsche Gesellschaft für Technische Zusammenarbeit. Berlin, Alemania. $335 \mathrm{p}$.

Lieberman, D., \& M. Lieberman. 1987. Forest tree growth and dynamics at La Selva, Costa Rica (1969-1982). J. Trop. Ecol. 3: 347-358

Manokaran N., \& K..M. Kochummen. 1987. Recruitment, growth and mortality of tree species in a lowland dipterocarp forest in Peninsular Malaysia. J. Trop. Ecol. 3: 315-330. 
Mata, R. 1997. Estudio detallado de suelos: Area de Demostración e Investigación La Tirimbina, Sarapiquí, Heredia, Costa Rica. Universidad de Costa Rica. Centro de Investigaciones Agronómicas (CIA). San José, Costa Rica. 52 p.

Martínez-Ramos, M., E. Alvarez-Buylla \& J. Sarukhán. 1989. Tree demography and gap dynamics in a tropical rain forest. Ecology 70: 555-558.

Popma, J. \& F. Bongers. 1988. The effect of canopy gaps on growth and morphology of seedling of rain forest species. Oecology 75: 623-632.

Poulson, T. \& W. Platt. 1989. Gap light regimes influence canopy tree diversity. Ecology 70: 553-555.

Quirós, D. \& Finegan, B. 1994. El manejo sustentable de un bosque natural tropical en Costa Rica: definición de un plan operacional y resultados de su aplicación. Colección silvicultura y manejo de bosques naturales no. 9. CATIE, Cartago, Costa Rica. 25 p.

Runkle, J.R. 1982. Patterns of disturbance in some oldgrowth mesic forests of eastern North America. Ecology 63: 1533-1546.

Swaine, M.D., D. Lieberman, \& F.E. Putz. 1987. The dynamics of tree populations in tropical forest: a review. J. Trop. Ecol. 3: 359-366.

Welden, C., S. Hewett, S. Hubbell \& R. Foster. 1991. Sapling survival, growth, and recruitment: Relationship to canopy height in a neotropical forest. Ecology 72: 35-50.

Whitmore, T.C. 1989. Canopy gaps and the two mayor groups of forest trees. Ecology 70: 536-538. 